\title{
Optimization of Depth-Based Routing for Underwater Wireless Sensor Networks through Intelligent Assignment of Initial Energy
}

\author{
Tanveer Ahmed ${ }^{1}$, Muhammad Kaleem ${ }^{* 1}$, Khurram Saleem Alimgeer ${ }^{1}$, Mustafa Shakir1 and Sajid Nazir ${ }^{2}$ \\ ${ }^{1}$ Department of Electrical Engineering, COMSATS Institute of Information Technology Islamabad, Pakistan
}

${ }^{2}$ Firstco Ltd., London, W2 6EU, UK

A R T I C L E I N F O

Article history:

Received: 29 May, 2017

Accepted: 20 July, 2017

Online: 05 October, 2017

Keywords:

Routing

Energy Efficiency

Wireless Sensor Networks

Network lifetime

Coverage Hole

\begin{tabular}{l} 
A B S T R A C T \\
\hline Underwater Wireless Sensor Networks (UWSNs) are extensively used to explore the diverse \\
marine environment. Energy efficiency is one of the main concerns regarding performance \\
of UWSNs. In a cooperative wireless sensor network, nodes with no energy are known as \\
coverage holes. These coverage holes are created due to non-uniform energy utilization by \\
the sensor nodes in the network. These coverage holes degrade the performance and reduce \\
the lifetime of UWSNs. In this paper, we present an Intelligent Depth Based Routing (IDBR) \\
scheme which addresses this issue and contributes towards maximization of network \\
lifetime. In our proposed scheme, we allocate initial energy to the sensor nodes according \\
to their usage requirements. This idea is helpful to balance energy consumption amongst \\
the nodes and keep the network functional for a longer time as evidenced by the results \\
provided.
\end{tabular}

\section{Introduction}

Underwater wireless sensor networks (UWSNs) have attained much attention nowadays due to their use in monitoring underwater environment. Underwater communication is characterized by larger propagation delay, high error rate and lower bandwidth. Acoustic signals are thus used for communications in underwater environment due to channel characteristics. Furthermore, nodes in UWSNs are powered with batteries that have limited lifetime. It is impractical to change the batteries of the nodes once deployed. The energy of the sensor nodes should be optimally used to avoid coverage holes i.e., nodes with no energy. Due to these reasons, routing in UWSNs is quite challenging and we need to address these issues while developing routing strategies for UWSNs [1].

A number of routing schemes are reported in literature to address the issue of efficient routing in UWSNs. One such protocol is depth based routing (DBR) protocol [2] which uses deployment depth of sensor nodes to determine the route of a data packet from its source node to the sink. Sensor nodes are usually deployed randomly in underwater environment and the sink is placed at the surface of water. Each sensor node is responsible to gather data and then send it to the sink in a cooperative manner. Some sensor

"Corresponding Author: Muhammad Kaleem, Department of Electrical Engineering, COMSATS Institute of Information Technology Islamabad, Pakistan Email: mkaleem@comsats.edu.pk

www.astesi.com

https://dx.doi.org/10.25046/aj0203219 nodes are deployed at a higher depth and some at a lower depth hence consituting a sensor network. Nodes with higher depth sense the required information and send it to a node present at lower depth in the form of data packets. Receiver nodes then send these packets to the next node located above. By repeating this process, data packets are passed from the sensor nodes to the sink which is placed at the water surface. It is important to note that nodes which are at a greater distance from the sink, only send their own data packets to low-depth nodes hence they consume lesser amount of energy and remain alive for a longer period of time. Whereas, sensor nodes that are near to the sink have to relay data packets received from deeper nodes in addition to sending their own data packets. So the nodes near the sink consume more energy than other nodes located deeper and thus die earlier. In this way an area with dead nodes i.e., a coverage hole is created in the network and no more data packets can be received at sink.

We propose an intelligent depth based routing (IDBR) protocol that overcomes coverage holes by assigning initial energy to the nodes in proportion to their distance from the sink. This work is an extended version of our previous work [3] in which we used idea of optimal energy assignment for improving lifetime of UWSNs and now we have enhanced this idea to make energy assignment more intelligent.

Rest of the paper is organized as follows. Section 2 gives an overview of the related work. Section 3 provides motivation for 


\section{T. Ahmed et al. / Advances in Science, Technology and Engineering Systems Journal Vol. 2, No. 3, 1799-1803 (2017)}

this research problem and we present our proposed protocol IDBR in Section 4. In section 5 performance of the proposed protocol is evaluated using simulations and Section 6 concludes the paper.

\section{Literature Review}

In this section, we present an overview of some of the protocols that have been reported in the literature to address the issues regarding efficient routing in UWSNs.

A hybrid protocol is presented in [4] to contribute towards balanced energy consumption in UWSNs. This technique is limited to the sparsely deployed networks only. Proposed scheme uses direct as well as hop-by-hop communication modes among sensor nodes during its operation. All nodes in the network are deployed linearly. Authors divide energy of each node into small chunks known as energy grades. When the protocol operation starts, all nodes are communicating in hop-by-hop mode. As nodes closer to the sink bear more data traffic, their energy falls earlier. Remaining energy grades determine the residual energy of a node. When the energy level of a low-depth node falls, it informs high depth sender nodes. Sender nodes then do not send their data packets to that node; instead they send it directly to the sink. This reduces data traffic load on the low-depth node and increases its lifetime. This process continues for all nodes and energy of the whole network is utilized in a balanced way.

Tayyaba et al. present another technique [5] for balanced energy consumption for UWSNs. The protocol is termed as depth based energy balanced hybrid (DB-EBH) protocol. In this protocol, all nodes are provided with equal amount of initial energy and then both direct and hop-by-hop communication modes are used to utilize the node energy in a balanced manner to maximize nodal lifetime. Sensor nodes in [4] are deployed linearly while in [5] these are deployed randomly in underwater environment.

Authors in [6] propose two energy balancing techniques for UWSNs. First technique is named as Efficient and Balanced Energy consumption Technique (EBET). EBET is based on the residual energy level of the nodes but instead of using direct communication mode authors propose route changes to reduce traffic load on receiving nodes. When residual energy level of some receiving node falls, it informs the sending node. Sending node does not send its data packet to that receiving node; instead it looks for some other receiving node with higher residual energy level and sends its data packet to it. In this way data traffic load on the nodes with lower residual energy is decreased and they remain alive for a longer period of time. Other technique called Enhanced Efficient and Balanced Energy consumption Technique (EEBET) uses the concept of depth threshold to enhance significance of the proposed technique.

EEDBR [7] is energy efficient version of DBR in which authors address limitations of DBR by suppressing transmission of extra data packets. Data packets are sent to the low-depth nodes and held for a short period of time called holding time. If the same packet is forwarded to the sink by some other node, its transmission is suppressed otherwise it is forwarded to the sink upon expiration of holding time. EEDBR performs better than DBR in terms of energy efficiency.

Another balanced energy consumption scheme is proposed by the authors in [8]. This protocol is based on optimal distance acquisition to achieve higher energy efficiency in Wireless Sensor Networks (WSNs). Authors in [9] and [10] also contribute towards balanced energy utilization in UWSNs using hybrid technologies with slightly different approaches from each other. H2-DAB [11] meets the challenges of UWSNs by implementing the dynamic addressing scheme among the sensor nodes without requiring the localization information. Another scheme known as RERP2R [12] uses the routing metric based on the physical distances between the sensor nodes and to achieve higher throughput in UWSNs. This protocol also gives the energy efficient solution for data forwarding along with better link quality. QELAR [13] is another routing scheme for UWSNs which strives to achieve longer network lifetime by selecting adequate receiving nodes for sender nodes. PULRP [14] introduces layered architecture and detailed algorithm to improve throughput and achieve energy efficiency in dense underwater conditions. HH-VBF [15] uses the vectors assumptions between the source and the destination nodes and proposes a vector-based algorithm to achieve low end-to-end delay in UWSNs.

\section{Motivation}

DBR uses deployment depth of the sensor nodes to perform routing operation. This fact makes it a very simple and easy to implement routing protocol. But DBR has a limitation that nodes deployed closer to the sink are out of energy after a short period of operation due to greater traffic load on them. This limitation causes creation of coverage holes in the network and data packets fail to reach the sink despite many alive nodes in the network. Coverge holes disrupt the network operation and available energy of the alive nodes cannot be utilised. Ideally, all nodes of the network should have lifetime closer to each other to avoid this energymismatch problem. EEDBR adresses energy wastage problem to some extent by suppressing extra transmissions. In EEDBR, nodes nearer to the sink are allocated with the same amount of energy as all other nodes. Ultimately these nodes die out because of greater load of data traffic. We address these limitations by proposing a more efficient scheme called IDBR which uses intelligent initial energy assignment to avoid coverage holes, improve throughput and prolong lifetime of the network.

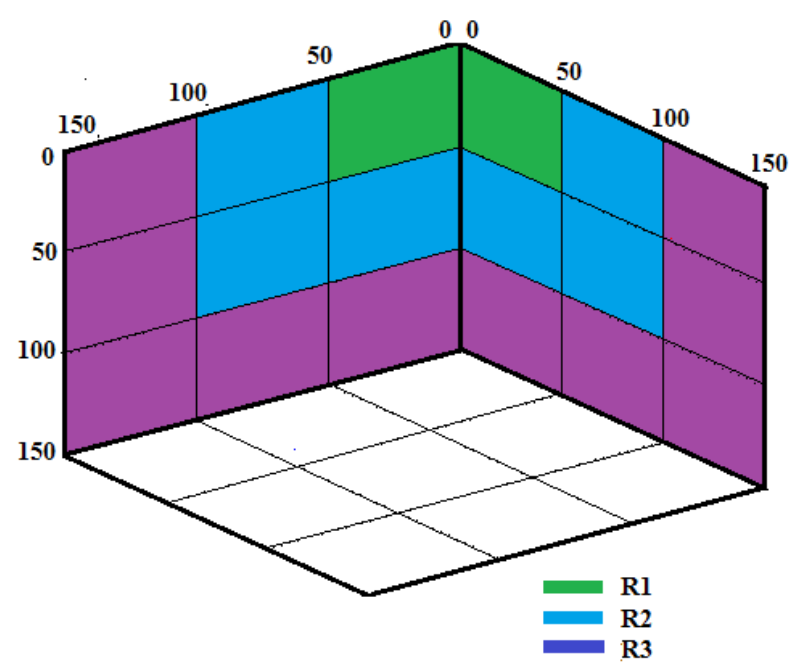

Figure 1. Network model 


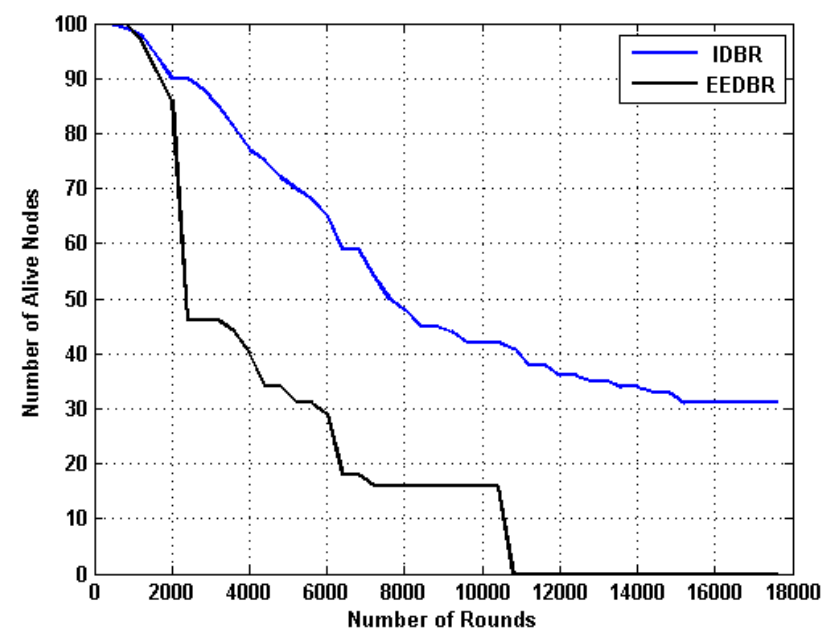

Figure 2. Comparison of alive nodes after each round for IDBR and EEDBR

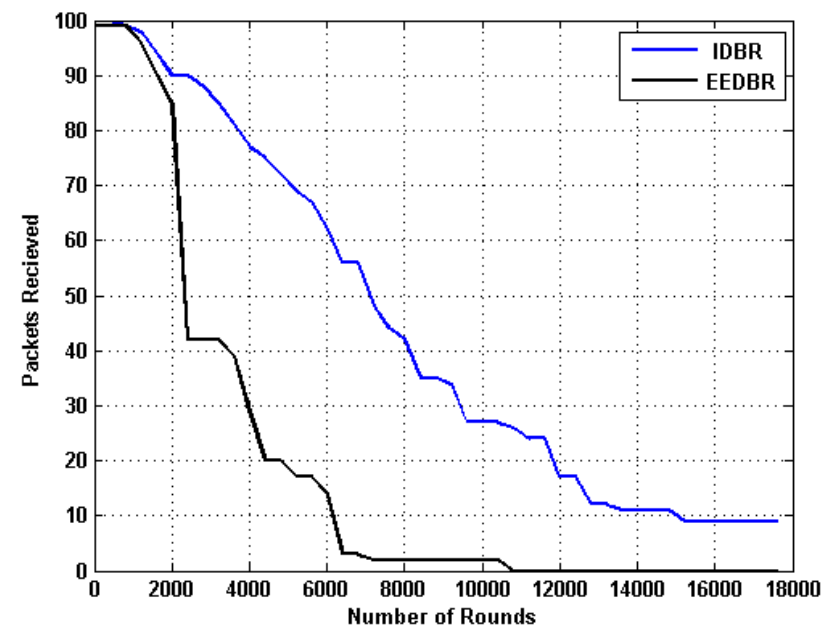

Figure 3. Comparison of packets received after each round in IDBR and EEDBR

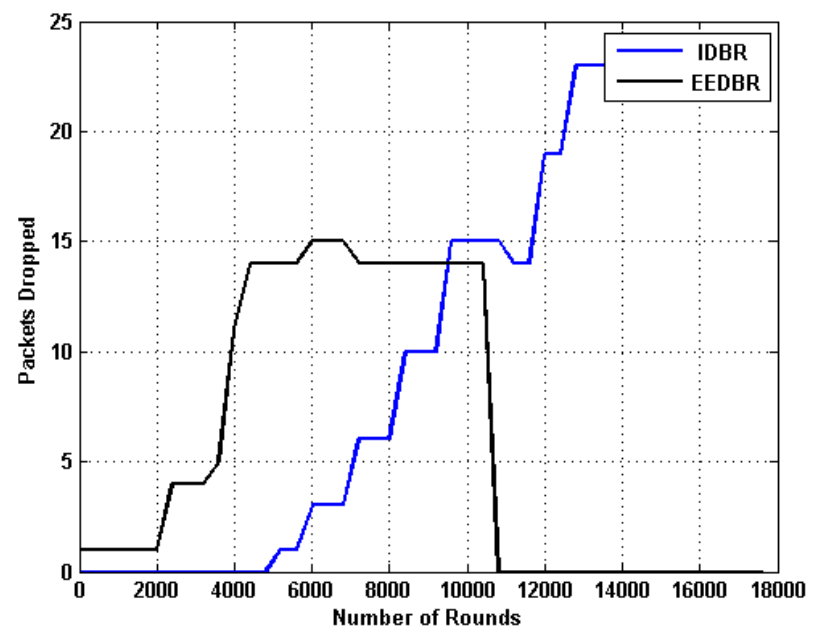

Figure 4. Comparison of packets dropped after each round for IDBR and EEDBR

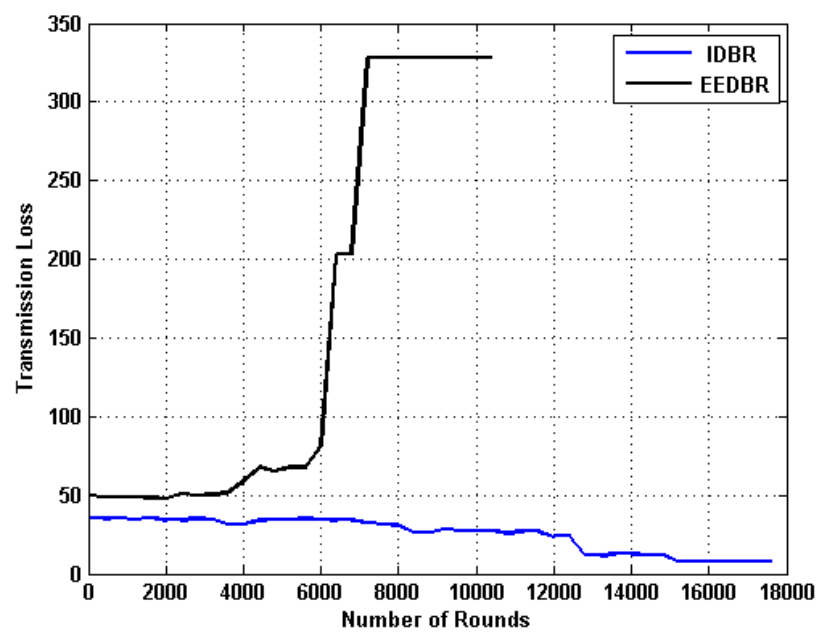

Figure 5. Comparison of transmission loss after each round for IDBR and EEDBR

\section{Proposed Scheme}

Energy efficiency is one of the most important issues in UWSNs. In this paper, we present an energy efficient routing protocol for UWSNs.

Figure 1 shows 3-Dimensional view of the network under consideration. It is assumed that nodes are deployed randomly in an area with equal length, width and depth.

In IDBR, we divide network area in three equal regions. Region nearest to the sink is named as R1. Second region is named as R2 at relatively greater distance from the sink compared to R1. Region at the maximum distance from the sink is named as R3. These regions are distinguished with the help of different colours in Figure 1.

Nodes belonging to R1 consume maximum amount of energy as they are responsible to receive and transmit data packets to the sink from all other nodes along with their own data packets. In IDBR, we assign maximum amount of initial energy to the sensor nodes belonging to R1. This assignments reflects the core idea of our scheme i.e allocation of initial energy keeping usage requirements of nodes in view.

Nodes belonging to R2 are assigned with relatively lesser amount of energy because they share lesser traffic load as compared to those nodes present in R1.

R3 nodes are provided with the least amount of initial energy as they have to send their own data packets only and hence utilize least amount of energy.

Total initial energies of IDBR and EEDBR are set be approximately equal to make realistic comparison between protocols. For IDBR, we assume that nodes in each region are one third of the total nodes. Equation (1) is used to realize this supposition:

$$
n / 3\left(E_{R 1}+E_{R 2}+E_{R 3}\right) \approx n E_{0}
$$

Where:

$n=$ Total number of nodes

$E_{R I}=$ Energy of each node in R1 for IDBR 


\section{T. Ahmed et al. / Advances in Science, Technology and Engineering Systems Journal Vol. 2, No. 3, 1799-1803 (2017)}

$E_{R 2}=$ Energy of each node in R2 for IDBR

$E_{R 3}=$ Energy of each node in R3 for IDBR

$E_{0}=$ Energy of each node for EEDBR

$E_{R 1}, E_{R 2}$ and $E_{R 3}$ are set in a way that equation (1) is satisfied. This relation ensures that total initial energies assigned to the entire network are approximately equal for both the protocols. The only difference is that; in EEDBR all nodes are assigned equal amount of energy but in IDBR nodes belonging to the different zones carry different initial energies. IDBR operation is summarized as follows:

i. After deployment, all nodes in the network need to know about depth information of each other. This is accomplished through exchange of a hello packet. Hello packet contains depth information of each node. When nodes exchange this packet, they obtain the depth information of each other. This step is repeated before each round of data transmission.

ii. Nodes that lie within the transmission range of a particular node, are known as neighbour nodes of that node. Each node in the network identifies its neighbor nodes when a hello packet is exchanged.

iii. A node which has a sensed data packet to send is known as a sender node. All sender nodes compare their own depth with the depth of their neighbor nodes. Sender node passes its data packet to a neighbor node for which two conditions demonstrated by inequalities (a) and (b) are satisfied. Neighbour node to whom the data packet is sent, is called receiver node.

$$
\begin{aligned}
& d_{r}<d_{s} \\
& d_{s}-d_{r}>d_{t h}
\end{aligned}
$$

where:

$d_{s}=$ Depth of sender node

$d_{r}=$ Depth of reciever node

$d_{t h}=$ Depth threshold

Inequalities (a) ensures that reciever node is a low-depth node so the data packet is being fowarded upwards i.e., towards the sink. While inequality (b) ensures that the distance between sender and reciever nodes is less than the depth threshold. Depth threshold is the minimum distance to which a node is allowed to send a data packet. Depth threshold makes sure that sender and reciever nodes are not too close. This idea avoids too many hops during the routing action of a data packet.

iv. Receiver nodes now become sender nodes for their lowdepth neighbors. Steps (ii) and (iii) are repeated until data packets are passed to the sink.

\section{Results and Discussion}

In this section, performance of proposed scheme IBDR is evaluated using simulations. Performance of IDBR is compared with that of EEDBR. Simulations are carried out using MATLAB. Length, width and depth of the network under consideration are set to be 150 meters each. Transmission range and depth thresholds are taken as 50 and 25 meters respectively. 100 nodes are deployed in the network and simulations are run for 18000 rounds of data transmission.

\section{Network Lifetime}

Network lifetime is defined as the time period from initialization of the network operation till death of the last node. In simulations, number of data transmission rounds determine network lifetime. More rounds of data transmission mean longer lifetime and vice versa. Number of alive nodes after each round for IDBR and EEDBR is shown in Figure 2.

It is clear from the graph that number of alive nodes in EEDBR drop to zero when around 11000 data transmission rounds are run. Whereas, in IDBR almost 30\% nodes are alive after 18000 data transmission rounds. This makes a huge difference between lifetimes of our proposed protocol IDBR and EEDBR. It is thus confirmed that intelligent assignment of initial energy to the nodes in IDBR increases operational lifetime of the network significantly as compared to that of EEDBR scheme.

\section{Throughput}

Data packets received at sink in unit time define throughput of a routing scheme. Comparison of data packets received after each round in IDBR and EEDBR is shown in Figure 3.

Figure 3 shows that throughput of IDBR is much better than that of EEDBR. In EEDBR number of received data packets falls drastically and no more packets are being received after 11000 data transmission rounds. While in IDBR, number of ecieved packets decreases rather smoothly and data packets are being received till the last round.

Data packets dropped after each round for both the protocols are compared in Figure 4. Number of packets dropped in EEDBR increases rapidly and finally drops to zero near 11000 rounds because at this stage no alive nodes are present in the network and no more data transmission is occurring. In IDBR, lesser data packets are being dropped than those of EEDBR. But more data packets are being dropped in IDBR during last rounds. The reason lies in the fact that more number of nodes in IDBR die with the passage of time.

\section{Energy Consumption}

One of the most important parameters to be considered in UWSNs is consumption of energy. Figure 5 shows comparison of transmission losses of IDBR and EEDBR.

Figure 5 shows that transmission loss in EEDBR is much greater than that of IDBR. Transmission loss of IDBR is almost constant during initial rounds but goes decreasing in last rounds because sensor nodes go on dying as the time passes. Transmission loss of EEDBR is much higher in the later rounds.

This comparison justifies the efficiency of IDBR in term of energy usage. Lesser and uniform energy consumption makes IDBR a much better routing protocol for UWSNs than EEDBR.

\section{Conclusion}

The paper compares the proposed protocol IDBR and EEDBR and shows significant improvement in lifetime, throughput and energy consumption of UWSNs. The intelligent assignment of initial energy to the sensor nodes enables us to distribute energy to the nodes according to their communications utilization. This idea improves the performance of depth based routing protocols for UWSNs and requires energy estimation of sensor nodes for realistic initial energy assignments. 


\section{References}

[1] F. Akyildiz, D. Pompili, and T. Melodia, "Underwater acoustic sensor networks: research challenges," Ad Hoc Networks 3, pp. 257279, 2005.

[2] Y. Hai, Z. J. Shi, and J. H. Cui, "DBR: depth-based routing for underwater sensor networks," International Conference on Research in Networking Springer Berlin Heidelberg, pp. 72-86, 2008.

[3] T. Ahmed, M. Chaudhary, M. Kaleem, and S. Nazir, "Optimized depth-based routing protocol for underwater wireless sensor networks." IEEE International Conference on Open Source Systems \& Technologies (ICOSST), pp. 147$150,2016$.

[4] L. Tayyaba, N. Javaid, S. M. Ali, M. Imran, and M. Alnuem, "Depth-Based Energy-Balanced Hybrid Routing Protocol for Underwater WSNs," International Conference on Intelligent Networking and Collaborative Systems (INCOS) Taiwan, pp. 262-267, 2015.

[5] L. Hanjiang, Z. Guo, K. Wu, F. Hong, and Y. Feng, "Energy balanced strategies for maximizing the lifetime of sparsely deployed underwater acoustic sensor networks," Sensors 9, vol. 9, pp. 6626-6651, 2009.

[6] J. Nadeem, M. Shah, A. Ahmad, M. Imran, M. I. Khan, and A. V. Vasilakos, "An Enhanced Energy Balanced Data Transmission Protocol for Underwater Acoustic Sensor Networks," Sensors 16, vol. 4, pp. 487, 2016.

[7] A. Wahid, S. Lee, H. Jeong, and Dongkyun Kim. "Eedbr: Energy-efficient depth-based routing protocol for underwater wireless sensor networks," Advanced Computer Science and Information Technology, Springer Berlin Heidelberg, pp. 223-234, 2011.

[8] L. Xuxun, "An optimal-distance-based transmission strategy for lifetime maximization of wireless sensor networks," IEEE Sensors Journal 15, vol. 6, pp. 3484-3491, 2015.

[9] D. Jinfeng, G. Zhang, Z. Guo, and J. Cao, "PAS: probability and sub-optimal distance-based lifetime prolonging strategy for underwater acoustic sensor networks," Wireless Communications and Mobile Computing 8, vol. 8, pp. 1061-1073, 2008.

[10] C. Jiabao, J. Dou, and S. Dong, "Balance transmission mechanism in underwater acoustic sensor networks," International Journal of Distributed Sensor Networks, pp. 2, 2015.

[11] M. Ayaz, A. Abdullah, I. Faye, and Y. Batira, "An efcient dynamic addressing based routing protocol for underwater wireless sensor networks", Computer Communications, vol. 35, no. 4, pp. 475486, 2012.

[12] A. Wahid, S. Lee, and D. Kim, "A reliable and energy-efficient routing protocol for underwater wireless sensor networks", International Journal of Communication Systems, pp.2048-2062, 2012.

[13] T. Hu and Y. Fei, "Qelar: a machine-learning-based adaptive routing protocol for energy-efcient and lifetime-extended underwater sensor networks", Mobile Computing, IEEE Transactions on, vol. 9, no. 6, pp. 796809, 2010.

[14] S. Gopi, G. Kannan, D. Chander, U. Desai, and S. Merchant, "Pulrp: Path unaware layered routing protocol for underwater sensor networks", IEEE International Conference on Communications, pp. 3141-3145, 2008.

[15] X. Peng, Z. Zhong, N. Nicolas, C. Jun-Hong, and S. Zhijie, "Efcient vectorbased forwarding for underwater sensor networks", EURASIP Journal on Wireless Communications and Networking, pp.195910, 2010. 